\title{
Hydrogenation Induced Structure and Property Changes in GdGa
}

Reji Nedumkandathil ${ }^{1}$, Verina F. Kranak ${ }^{1}$, Robert Johansson ${ }^{2}$, Jonas Ångström ${ }^{3}$, Oliver Balmes $^{4}$, Mikael S. Andersson ${ }^{5}$, Per Nordblad ${ }^{5}$, Ralph H. Scheicher ${ }^{2}$, Martin Sahlberg ${ }^{3}$, and Ulrich Häussermann ${ }^{1}$

${ }^{1}$ Department of Materials and Environmental Chemistry, Stockholm University, SE-10691 Stockholm, Sweden

${ }^{2}$ Department Physics and Astronomy, Uppsala University, Box 516, SE-75210 Uppsala, Sweden

${ }^{3}$ Department of Chemistry - Ångström Laboratory, Uppsala University, Box 538, SE-75121

Uppsala, Sweden

${ }^{4}$ MAX IV Laboratory, Lund University, Box 118, SE-22100 Lund, Sweden

${ }^{5}$ Department of Engineering Sciences, Uppsala University, Box 534, SE-75121 Uppsala, Sweden

\begin{abstract}
Hydrides $\mathrm{GdGaH}_{\mathrm{x}}$ were obtained by exposing the Zintl phase GdGa with the CrB structure to a hydrogen atmosphere at pressures from 1.5 to 50 bar and temperatures from 50 to $500{ }^{\circ} \mathrm{C}$. Structural analysis by powder $\mathrm{X}$-ray diffraction suggests that conditions with hydrogen pressures in a range between 15 and 50 bar and temperatures below $500{ }^{\circ} \mathrm{C}$ afford a uniform hydride phase with the $\mathrm{NdGaH}_{1.66}$ structure (Cmcm, $a=3.9867(7) \AA, b=12.024(2) \AA, c=$ 4.1009(6) $\AA$ ) which hosts $\mathrm{H}$ in two distinct positions, H1 and H2. H1 is coordinated in a tetrahedral fashion by $\mathrm{Gd}$ atoms, whereas $\mathrm{H} 2$ atoms are inserted between $\mathrm{Ga}$ atoms. The assignment of the $\mathrm{NdGaH}_{1.66}$ structure is corroborated by first principles DFT calculations. Modeling of phase and structure stability as a function of composition resulted in excellent agreement with experimental lattice parameters when $\mathrm{x}=1.66$ and revealed the presence of five-atom moieties Ga-H2-Ga-H2-Ga in $\mathrm{GdGaH}_{1.66}$. From in situ powder X-ray diffraction using synchrotron radiation it was established that hydrogenation at temperatures above 200 ${ }^{\circ} \mathrm{C}$ affords a hydride with $\mathrm{x} \approx 1.3$, which is stable up to $500{ }^{\circ} \mathrm{C}$, and that additional $\mathrm{H}$ absorption, yielding $\mathrm{GdGaH}_{1.66}$, takes place at lower temperatures. Consequently, $\mathrm{GdGaH}_{1.66}$ desorbs $\mathrm{H}$ above $\mathrm{T}=200{ }^{\circ} \mathrm{C}$. Without the presence of hydrogen, hydrides $\mathrm{GdGaH}_{\mathrm{x}}$ decompose at temperatures above $300{ }^{\circ} \mathrm{C}$ into $\mathrm{GdH}_{2}$ and an unidentified $\mathrm{Gd}-\mathrm{Ga}$ intermetallics. Thus the hydrogenation of GdGa is not reversible. From magnetic measurements the CurieWeiss constant and effective magnetic moment of $\mathrm{GdGaH}_{1.66}$ were obtained. The former indicates antiferromagnetic interactions, the latter attains a value of $\sim 8 \mu_{\mathrm{B}}$ which is typical for compounds containing $\mathrm{Gd}^{3+}$ ions.
\end{abstract}




\section{Introduction}

The great majority of rare earth (RE) metals form monogallides REGa which all crystallize with the orthorhombic $\mathrm{CrB}$ structure featuring linear zigzag chains of $\mathrm{Ga}$ atoms (Figure 1a) [1-3]. The REGa compounds display interesting magnetic properties and have recently attracted attention due to their potential as magnetocaloric materials [4-10]. In general they are ferromagnets and across the lanthanide series the Curie temperature $\mathrm{T}_{\mathrm{C}}$ increases from 32 $\mathrm{K}$ for PrGa to a maximum of $\sim 190 \mathrm{~K}$ for $\mathrm{GdGa}$ and then decreases to $15 \mathrm{~K}$ for TmGa [11-13]. On cooling, ferromagnetically ordered REGa compounds undergo spin reorientation transitions $[4,7,8,14]$.

The ferromagnetic properties of REGa are a consequence of the coupling of magnetic moments arising from localized $4 \mathrm{f}$ electrons, which is mediated via conduction electrons according to the Ruderman-Kittel-Kasuya-Yosida (RKKY) coupling mechanism [15]. First principles calculations based on the RKKY interaction reproduce well observed ferromagnetic transition temperatures [16]. The validity of the RKKY coupling mechanism, however, contradicts the simple description of REGa compounds as Zintl phases [17]. Following the Zintl concept and assuming trivalent $\mathrm{RE}, \mathrm{Ga}$ will correspond to six-electron species $\mathrm{Ga}^{3-}$, which are connected to polyanionic zigzag chains via single bonds. Within this simple picture all valence electrons are then localized as bonds and lone pairs, i.e. a charge balanced Zintl phase $\mathrm{RE}^{3+} \mathrm{Ga}^{3-}$ with singly bonded chains would not leave any excess electrons for mediating ferromagnetic coupling.

To reconcile RKKY coupling within the Zintl concept one may assume that Ga is just reduced to $\mathrm{Ga}^{2-}$ species, now forming $\pi$-bonded zigzag chains in a charge imbalanced (electron excess) Zintl phase $\mathrm{RE}^{3+} \mathrm{Ga}^{2-} \mathrm{e}^{-}$. Hydrogen incorporation in REGa represents a possibility to probe the electronic structure of REGa systems and, thus, to provide insight into the interplay between chemical bonding in the polyanion and magnetic properties. In particular, itinerant electrons may be localized as $\mathrm{H}^{-}$through the formation of interstitial hydrides [18,19]. This should express in a change of the strength and possibly also the sign of the magnetic interaction. Provided that hydrogen uptake and desorption are easily reversible, one could even envision switchable magnetic properties with systems REGa/ $\mathrm{REGaH}_{\mathrm{x}}$.

Previously we explored the structure and magnetic property changes of $\mathrm{NdGa}$ upon hydrogenation [20]. We found that $\mathrm{NdGa}$ transforms into a hydride $\mathrm{NdGa}_{1.66}$ by incorporating $\mathrm{H}$ into two distinct positions, $\mathrm{H} 1$ and $\mathrm{H} 2$. Interstitial $\mathrm{H} 1$ atoms are exclusively coordinated by $\mathrm{RE}$ atoms, whereas $\mathrm{H} 2$ atoms are inserted between $\mathrm{Ga}$ atoms and part of a two-dimensional $\mathrm{Ga}-\mathrm{H}$ polyanion (Figure 1b). At the same time the magnetic interaction changed from ferromagnetic to antiferromagnetic nature. In this paper we report on the hydrogenation behavior of $\mathrm{GdGa}$ which possesses the highest $\mathrm{T}_{\mathrm{C}}$ among REGa compounds. 


\section{Methods}

1. Synthesis. GdGa was synthesized by arc-melting mixtures of pieces of Gd (99.99\%, SigmaAldrich) and Ga (99.999\%, Sigma-Aldrich) metal in a glove box. A slight excess of Gd (2\%) was applied. Sample batches corresponded to $0.5-1.0 \mathrm{~g}$ in total mass. To ensure homogeneity, samples were re-melted five times and flipped between each melting. The total mass loss was less than $1 \%$. Arc-melted GdGa samples were then heat-treated at $900{ }^{\circ} \mathrm{C}$ in an evacuated fused silica ampoule for 10 - 20 days and subsequently ground for hydrogenation experiments. Hydrogenous $\mathrm{GdGa}$ was produced by exposing $\mathrm{GdGa}$ in an autoclave to a $\mathrm{H}_{2}$ atmosphere using temperatures from 50 to $600{ }^{\circ} \mathrm{C}$, pressures from 1.5 to $80 \mathrm{bar}$, and dwelling times from $15 \mathrm{~min}$ to $20 \mathrm{~h}$. GdGa and its hydrides appear stable in air.

2. Structural Characterization of $G d G a$ and hydrogenous $G d G a$. Powder X-ray diffraction (PXRD) patterns of GdGa and hydrogenated GdGa samples were recorded on a Panalytical X'Pert Pro diffractometer in $\theta-2 \theta$ diffraction geometry employing $\mathrm{Cu} K_{\alpha}$ radiation $(\lambda=1.5418$ $\AA$ ). For room temperature measurements powder samples were mounted on a Si wafer zerobackground holder and diffraction patterns were measured in a $2 \theta$ range $5-90^{\circ}$. For high temperature PXRD studies powder samples were heated in dynamic vacuum $\left(\sim 10^{-5}\right.$ bar $)$ to $450{ }^{\circ} \mathrm{C}$ using an Anton Paar XRK 900 chamber equipped with Be windows and connected to a temperature controller. Data in a $2 \theta$ range $20-50^{\circ}$ were collected from $100{ }^{\circ} \mathrm{C}$ in steps of $50{ }^{\circ} \mathrm{C}, 20 \mathrm{~min}$ acquisition time and $3{ }^{\circ} \mathrm{C} / \mathrm{min}$ heating rate between the steps. The Rietveld method [21] as implemented in the FullProf program [22] was used for structure and phase analysis. Structure refinements were based on the established structure models for GdGa [2] and $\mathrm{NdGaH}_{1.66}[20]$.

3. In-situ diffraction. The hydrogen uptake of GdGa was studied in situ by PXRD using synchrotron radiation at the I711 beam line $(\lambda=0.991 \AA)$ at the MAX-IV laboratory [23]. A small amount of powder sample was placed in a fused silica capillary which was in turn placed in a single crystal sapphire tube mounted in a sample cell. This cell is dedicated for in situ studies using high gas pressures, high temperatures as well as magnetic fields [24, 25]. The sample was subjected to 20 bar $\mathrm{H}_{2}$ after being flushed three times with high purity Ar. Subsequently the sample was heated with a constant rate from room temperature to $500{ }^{\circ} \mathrm{C}$ using a Kanthal resistive wire coiled around the sapphire tube and then cooled to room temperature. During the experiment a series of diffractograms were recorded using a Titan area CCD detector. The resulting data was reduced from 2D images to $1 \mathrm{D}$ diffractograms using the FIT2D software [26]. Sequential Rietveld refinement with the FullProf program [22] was performed to obtain the lattice parameters as a function of time (and temperature). The background was refined as a six-coefficient polynomial and the peaks were described by a Lorentzian function. After fitting the background, peak function, lattice parameters and scale factor to the first pattern the sequential refinement was performed. During the sequential refinement nine parameters were refined: five background parameters; the $a, b$ and $c$ lattice parameters and the scale factor.

4. Computations. The theoretical calculations of the electronic structure and total energies of $\mathrm{GdGa}$ and structure models for $\mathrm{GdGaH}_{\mathrm{x}}(1 \leq \mathrm{x} \leq 2)$ were performed using the first-principles 
all-electron projector augmented waves (PAW) method [27,28] within the Vienna ab initio Simulation Package (VASP) [29,30]. Exchange-correlation effects were treated within the generalized gradient approximation (GGA) via the Perdew-Burke-Ernzerhof parametrization PBE [31]. A conjugate gradient algorithm was used to relax the atomic nuclei positions to a local minimum in the total energy landscape. Structure relaxations for $\mathrm{GdGaH}_{\mathrm{x}}$ did not employ any symmetry restrictions. The findsym program [32] was used to check for symmetry of atom arrangements after relaxations. Forces were converged to better than $10^{-}$ $3 \mathrm{eV} / \mathrm{A}$. The plane wave basis set was terminated at a kinetic energy cutoff of $600 \mathrm{eV}$. Partial occupancy of hydrogen was accounted for in a $3 \times 1 \times 1$ supercell based on the orthorhombic $\mathrm{CrB}$ type structure with $\mathrm{Z}=12$ and considering the compositions $\mathrm{GdGaH}_{1.33}, \mathrm{GdGaH}_{1.50}$, $\mathrm{GdGaH}_{1.66}$. The number of calculations of random $\mathrm{H}$ distributions was large enough so that convergence was reached in the total energy, i.e. adding more calculations of random distributions made insignificant difference to the mean total energy. Stoichiometric GdGa, GdGaH and $\mathrm{GdGaH}_{2}$ were calculated both within a $3 \times 1 \times 1$ supercell and a single unit cell $(\mathrm{Z}=4)$. The heat of formation, referring to zero Kelvin, is given by:

$$
\Delta \mathrm{H}=1 / \mathrm{x}\left[\left(\mathrm{E}\left(\mathrm{GdGaH}_{\mathrm{x}}\right)-\mathrm{E}(\mathrm{GdGa})-\mathrm{x} / 2 \mathrm{E}\left(\mathrm{H}_{2}\right)\right](1 \leq \mathrm{x} \leq 2)\right.
$$

where $\mathrm{E}$ denotes the total energy of the in brackets enclosed system. The number of $\mathrm{H}$ atoms in the calculation is denoted by $\mathrm{x}$. For the $\mathrm{H}_{2}$ molecule, a box of $8 \times 8 \times 8 \AA^{3}$ dimensions was used with $\Gamma$-point sampling of the Brillouin zone. Electronic structure calculations for single unit cells used a grid of $21 \times 7 \times 21 \mathrm{k}$-points to sample the Brillouin zone [33]. For this work theoretical calculations were used for assessing structural variations and enthalpy changes upon $\mathrm{H}$ incorporation. As both $\mathrm{GdGa}$ and its hydrides do not show magnetic correlations at temperatures above $200 \mathrm{~K}$ the contribution of magnetic coupling to total energies was assumed negligible. Hence, for simplicity, all systems were treated as non-magnetic and calculations were thus non spin-polarized.

5. Magnetic Measurements. Magnetization measurements were performed as a function of temperature using a Quantum Design MPMS SQUID magnetometer. M vs. T measurements were done both at low field $(\mathrm{H}=4 \mathrm{kA} / \mathrm{m})$ in order to determine the transition temperature of the samples, as well as in high field $(\mathrm{H}=80 \mathrm{kA} / \mathrm{m})$ in order to do a Curie-Weiss fit and estimate the effective number of bohr magnetons $\left(\mu_{\mathrm{eff}}\right)$ and Curie-Weiss temperature $\left(\Theta_{\mathrm{CW}}\right)$ of the samples. M vs. $\mathrm{H}$ measurements were performed using a Quantum Design PPMS VSM magnetometer at $6 \mathrm{~K}$ in the field range of $\pm 7200 \mathrm{kA} / \mathrm{m}$ to determine the saturation magnetization as well as study the field dependence of the samples.

\section{Results and Discussion}

\section{A. Hydrogenation behavior of GdGa.}

GdGa was exposed to a hydrogen atmosphere at various conditions in order to explore temperature, pressure and time aspects of the hydrogenation reaction. Figure 2 shows selected PXRD patterns of reaction products when varying temperature and pressure, respectively, and 
keeping dwelling time constant $(2 \mathrm{~h})$. GdGa is remarkably susceptible to $\mathrm{H}$ uptake. As a matter of fact, kinetic barriers appear to be small. Quantitative hydride formation is observed at lowest $\mathrm{T}$ in the temperature series $\left(\sim 50{ }^{\circ} \mathrm{C}, 20\right.$ bar $)$ and lowest $\mathrm{p}$ in the pressure series $(\sim 1.5$ bar, $100{ }^{\circ} \mathrm{C}$ ). The great similarity of the PXRD patterns of obtained products from reactions performed between 50 and $400{ }^{\circ} \mathrm{C}$ and at a pressure of 20 bar suggests that a uniform hydride was obtained. At higher temperatures (above $500{ }^{\circ} \mathrm{C}$ ) $\mathrm{GdH}_{2}$ can be identified in the PXRD, which indicates $\mathrm{H}$-induced oxidative decomposition of $\mathrm{GdGa}$ [18]. When varying pressure (and keeping annealing temperature constant at $100{ }^{\circ} \mathrm{C}$ ) the hydride seen in the temperature series is produced between 15 and 50 bar. At lower pressures differences in the PXRD patterns indicate the formation of different ("intermediate") hydrides. Similar to high temperatures, high hydrogen pressures ( 80 bar) result in a decomposition of $\mathrm{GdGa}$ (formation of $\mathrm{GdH}_{2}$ ).

The diffraction patterns of the temperature series can be indexed in a $C$-centered orthorhombic cell with lattice parameters $a \approx 3.99 \AA, b \approx 12.02 \AA, c \approx 4.10 \AA$ resembling the ones of the starting material GdGa ( $a=4.340(3) \AA, b=11.012(2) \AA, c=4.105(3) \AA$ (cf. Figure $\mathrm{S} 1$ in the supporting information)). This is similar to $\mathrm{NdGa}$ for which hydrogenation leads to a phase $\mathrm{NdGaH}_{1.66}$ with lattice parameters closely related to the starting material. As a matter of fact, the PXRD patterns of the uniform hydride product can be satisfactorily fit to the $\mathrm{NdGaH}_{1.66}$ structure model (Figure 3a). When applying it to the data of "intermediate" hydrides obtained at $\mathrm{H}_{2}$ pressures below 15 bar unindexed peaks remain (Figure $3 \mathrm{~b}$ ), which may be accounted for by doubling the $a$ or $c$ axis (or both) with respect to the original $C$-centered orthorhombic cell. Results of the Cmcm structure refinements are compiled in Table 1.

In conclusion, the $\mathrm{T}$ and $\mathrm{p}$ dependent hydrogenation reactions with $\mathrm{GdGa}$ suggest great similarities to earlier investigated $\mathrm{NdGa}$ [20]. We assign the hydride phase yielding a $C$ centered orthorhombic PXRD pattern to $\mathrm{GdGaH}_{1.66}$ - in analogy with $\mathrm{NdGa}$. This assignment is strongly supported by DFT simulations of $\mathrm{GdGaH}_{\mathrm{x}}$ structures with $\mathrm{x}>1$ (see below). We note that the exceptionally high neutron capture cross section of Gd prohibits the application of neutron diffraction for structural analysis of hydrogenous GdGa.

To investigate the time aspect of GdGa hydrogenations we performed an in situ PXRD experiment where $\mathrm{GdGa}$ was heated to $500{ }^{\circ} \mathrm{C}$ in a hydrogen atmosphere of 20 bar at a rate of $10{ }^{\circ} \mathrm{C} / \mathrm{min}$ and subsequently cooled. The results are compiled in Figure 4. Upon heating PXRD patterns changed abruptly after $35 \mathrm{~min}\left(\sim 370{ }^{\circ} \mathrm{C}\right)$, and more subtly and gradually upon cooling at temperatures below $200{ }^{\circ} \mathrm{C}$ (Figure $4 \mathrm{a}$ ). The diffraction patterns were indexed in a $C$-centered orthorhombic cell and Figure $4 \mathrm{~b}$ shows the relative change of the cell parameters with respect to the starting material GdGa. The structure change upon heating corresponds to an expansion of the $b$ axis and a contraction of the $a$ axis by 7.5 and $6.5 \%$, respectively. (At $500{ }^{\circ} \mathrm{C}$ diffraction peaks from $\mathrm{GdH}_{2}$ appear, which is consistent with the observation from exsitu investigations, see above). The structure change upon cooling corresponds to a further increase of $b /$ decrease of $a$. During heating and cooling the $c$ parameter varies only very slightly. In Figure $4 \mathrm{~b}$ are inserted the values from DFT simulations for phases $\mathrm{GdGaH}_{1.0}$, $\mathrm{GdGaH}_{1.33}$ and $\mathrm{GdGaH}_{1.66}$. It can be recognized that there is a close correspondence of the 
parameters of the product after cooling (which is identical to that obtained from ex-situ experiments at 20 bar $\mathrm{H}_{2}$ ) with the ones of the simulated $\mathrm{GdGaH}_{1.66}$ structure.

There are two important observations with the in situ experiment: (i) Hydrogenation proceeds already at low temperatures (as seen in the gradual change of lattice parameters) and above $\sim 350{ }^{\circ} \mathrm{C}$ kinetic barriers are absent (abrupt change of lattice parameters). Hydrogen absorption at these (high) temperatures leads to the formation of a hydride phase $\mathrm{GdGaH}_{\sim 1.3}$ which is stable to up to $500{ }^{\circ} \mathrm{C}$ (onset of decomposition). (ii) At lower temperatures (below $200{ }^{\circ} \mathrm{C}$ ) additional $\mathrm{H}$ is absorbed leading to a hydride $\mathrm{GdGaH}_{1.66}$, which is also the final product in synthesis experiments using $\mathrm{H}_{2}$ pressures above 15 bar. Consequently, $\mathrm{GdGaH}_{1.66}$ is thermally labile. When heating $\mathrm{GdGaH}_{1.66}$ in dynamic vacuum $\left(\sim 10^{-5}\right.$ bar $)$ and recording simultaneously PXRD patterns, changes are apparent already at $100{ }^{\circ} \mathrm{C}$ (see supporting information, Figure S2). At temperatures above $300{ }^{\circ} \mathrm{C}$ hydrogenous $\mathrm{GdGa}$ decomposes into $\mathrm{GdH}_{2}$ and a poorly crystalline $\mathrm{Gd}-\mathrm{Ga}$ intermetallics which could not be identified. Thus, in contrast with $\mathrm{NdGa}$ the hydrogenation of $\mathrm{GdGa}$ is not reversible. It is clear that the hydride phase $\mathrm{GdGaH}_{\mathrm{x}}(\mathrm{x}=1$ - 1.33) seen in the in situ experiment between 200 and $500{ }^{\circ} \mathrm{C}$ (in the presence of $\mathrm{H}_{2}$ ) cannot be obtained from desorption experiments. We also observed that $\mathrm{GdGaH}_{1.66}$ samples lose $\mathrm{H}$ over time when storing at room temperature (see supporting information, Figure S3).

\section{B. Structural variations and energetics of $\mathbf{H}$ incorporation}

In the orthorhombic $\mathrm{CrB}$ type structure of $\mathrm{GdGa}$ (space group $\mathrm{Cmcm}, \mathrm{Z}=4$ ) both $\mathrm{Gd}$ and $\mathrm{Ga}$ atoms are situated on sites $4 \mathrm{c}(0, \mathrm{y}, 1 / 4)$. Ga atoms center $\mathrm{Gd}_{6}$ trigonal prisms and form polyanionic zigzag chains running along [001]. The arrangement of Gd atoms in "Ga-free" layers corresponds to an array of edge sharing tetrahedra (cf. Figure 1). The center of these tetrahedra, which also corresponds to a $4 \mathrm{c}$ site, is occupied by $\mathrm{H}$ in the $\mathrm{NdGaH}_{1.66}$ structure. Its structure was unambiguously characterized from powder neutron diffraction data (Figure 1b). The tetrahedral, interstitial, $\mathrm{H}$ position was termed $\mathrm{H} 1$. Additional $\mathrm{H}(\mathrm{H} 2)$ is incorporated between $\mathrm{Ga}$ atoms of neighboring chains. The $\mathrm{H} 2$ position is partially occupied ( $2 / 3)$. The implications of two chemically different $\mathrm{H}$ for $\mathrm{NdGaH}_{1.66}$, interstitial $\mathrm{H} 1$ primarily leading to an oxidation of the Ga polyanion and $\mathrm{H} 2$ being part of a novel two-dimensional polyanion $[\mathrm{GaH}]^{2-}$, have been described in detail [20].

For structure analysis of hydrogenous GdGa neutron diffraction cannot be applied. However, the structural consequences of $\mathrm{H}$ incorporation in $\mathrm{GdGa}$ can be assumed to be very similar to $\mathrm{NdGa}$. In order to corroborate this (and also to obtain insight into the distribution of $\mathrm{H}$ in hydrogenous $\mathrm{GdGa}$ ) structure and phase stability of various compositions $\mathrm{GdGaH}_{\mathrm{x}}(\mathrm{x} \geq 1)$ were investigated by DFT calculations. The salient results are presented in Table 2 and as supporting information, Table S1. First we note that the $\mathrm{Cmcm}$ structure of $\mathrm{GdGa}$ is excellently reproduced by DFT structure optimization (cf. Tables 1, 2, S1), which validates the use of non spin-polarized calculations for this purpose. The occupation of the $\mathrm{H} 1$ position is energetically preferred over $\mathrm{H} 2$ (by $0.43 \mathrm{eV}$ per formula unit $\mathrm{GdGaH}$ ) and, thus, is assumed to represent the first step in the hydrogenation of $\mathrm{GdGa}$. The transition from $\mathrm{GdGa}$ to $\mathrm{GdGaH}$ with the $\mathrm{H} 1$ position filled is accompanied with a shortening of the $a$ and an elongation of the $b$ axis by 7.2 and $6.0 \%$, respectively. This trend proceeds gradually when filling the $\mathrm{H} 2$ 
position up to $\mathrm{x}=0.66$. At the same time the $c$ parameter (which coincides with the direction of the Ga zigzag chains in the $\mathrm{Cmcm}$ structure) changes very little. As already mentioned, the calculated changes of the lattice parameters compare well with the observation of the in situ PXRD experiment. It is interesting to note that the associated changes of the unit cell volume are minute. As a matter of fact, $\mathrm{GdGaH}_{1.66}$ attains virtually the same unit cell volume as the starting material GdGa. Upon completely filling of the $\mathrm{H} 2$ position (and thus arriving at a hydride composition $\mathrm{GdGaH}_{2}$ ) the trend in the cell parameter changes is reversed, i.e. when going from $\mathrm{x}=1.66$ to 2 the $a$ parameter increases and the $b$ parameter decreases.

Figure 6 shows the evolution of structures when going from $x=1$ to 1.66 according to DFT modeling. $\mathrm{GdGaH}$ with a fully occupied $\mathrm{H} 1$ position $(\mathrm{x}=1)$ was described with a single unit cell $(Z=4)$. Partial occupancy of the $\mathrm{H} 2$ position $(x>1)$ was simulated by model structures using a $3 \times$ a supercell containing 12 formula units. For $\mathrm{x}=1.33$ the most stable arrangement corresponds to $\mathrm{H} 2$ atoms connecting two neighboring Ga zigzag chains into three-atom $\mathrm{Ga}-\mathrm{H}$ Ga strands. H2 atoms are symmetrically located between two Ga atoms at a distance $2.11 \AA$. In addition, this structure model contains original Ga-Ga zigzag chains. The ratio between zigzag chains and three-atom strands is $1: 1$. In the most stable arrangement for $\mathrm{x}=1.66 \mathrm{H} 2$ atoms bridge all $\mathrm{Ga}$ zigzag chains into five-atom strands $\mathrm{Ga}-\mathrm{H}-\mathrm{Ga}-\mathrm{H}-\mathrm{Ga}$. Here $\mathrm{H} 2$ atoms are asymmetrically located between two Ga atoms at distances 1.92 and $2.33 \AA$ A. (For more details see supporting information, Table S1). The calculated powder pattern for this $\mathrm{GdGaH}_{1.66}$ model (Figure 6b) matches very well the experimental pattern of the uniform hydride product obtained from hydrogenations using pressures above 15 bar (Figure 2, 3a).

The electronic density of states (DOS) of stoichiometric $\mathrm{GdGa}, \mathrm{GdGaH}$ and $\mathrm{GdGaH}_{2}$ is shown as supporting information, Figure S3.The energy of formation of $\mathrm{GdGaH}_{\mathrm{x}}$ according to $\mathrm{GdGa}$ $+\mathrm{x} / 2 \mathrm{H}_{2}$ is shown in Figure 7 and compared with $\mathrm{NdGa}+\mathrm{x} / 2 \mathrm{H}_{2}$. Upon filling the $\mathrm{H} 2$ position (i.e. for $1<\mathrm{x} \leq 2$ ) its magnitude decreases in a linear fashion with increasing $\mathrm{x}$. This is similar to $\mathrm{NdGaH}_{\mathrm{x}}$ but values for $\mathrm{GdGaH}_{\mathrm{x}}$ are lower. This may relate to its different desorption behavior. $\mathrm{NdGaH}_{1.66}$ desorbs $\mathrm{H}$ in two distinct steps, at around 250 and $450{ }^{\circ} \mathrm{C}$, after which $\mathrm{CrB}$-type $\mathrm{NdGa}$ is quantitatively reformed [20]. In contrast, although $\mathrm{GdGaH}_{1.66}$ starts desorbing $\mathrm{H}$ below $200{ }^{\circ} \mathrm{C} \mathrm{GdGa}$ is not obtained by thermal decomposition. It appears likely that in the early desorption products both $\mathrm{H} 1$ and $\mathrm{H} 2$ positions are partially occupied. The same may be the case for products from hydrogenations at low $\mathrm{p}$ (below 15 bar, i.e. the intermediate hydrides). The diffraction patterns of hydrogenous GdGa from both processes are similar (cf. Figure 2b, Figures S2 and S3).

\section{H-induced magnetic property changes}

GdGa is known to be ferromagnetic with a reported $\mathrm{T}_{\mathrm{C}}$ of $\sim 190 \mathrm{~K}$ and a spin reorientation at $85 \mathrm{~K}[4,14,16,33,34]$. A similar behavior is confirmed in our sample; Figure 7a shows zero field cooled (ZFC) and field cooled (FC) magnetization vs. temperature curves of GdGa measured in an applied field of $4 \mathrm{kA} / \mathrm{m}$. The sharp rise of the magnetization at about $180 \mathrm{~K}$ indicates the ferromagnetic transition and the peak close to $70 \mathrm{~K}$ reflects the spin reorientation. There is a substantial change in the behavior of the magnetization after hydrogenation, as seen in Figure $7 b$ (which shows ZFC and FC magnetization vs. temperature 
curves for $\mathrm{GdGaH}_{1.66}$ in an applied field of $4 \mathrm{kA} / \mathrm{m}$ ) indicating an antiferromagnetic transition by the sharp maxima at about $45 \mathrm{~K}$. The separation between the ZFC and FC curves at temperatures below the transition temperature reveals a weak excess moment due to formation of spin domains with residual ferromagnetic components. In order to fit the behavior at higher temperatures to the Curie-Weiss law $\left(\mathrm{C} /\left(\mathrm{T}-\theta_{\mathrm{CW}}\right)\right)$, data for both samples were also recorded at a higher field, $80 \mathrm{kA} / \mathrm{m}$. The derived Curie-Weiss constant $\theta_{\mathrm{CW}}$ and the effective number of bohr magnetons $\left(\mu_{\text {eff }}\right.$ ) are tabulated in Table 3. Both $\mathrm{GdGa}$ and $\mathrm{GdGaH}_{1.66}$ have an effective magneton number close to 8 , which is the expected number for $\mathrm{Gd}^{3+}$. As in the case of $\mathrm{NdGa}$ [20] there is a change of sign of $\theta_{\mathrm{CW}}$ after hydrogenation, again indicating that GdGa transforms from a ferromagnet to an antiferromagnet upon hydrogenation. The full hysteresis curve of GdGa plotted in Figure 7c shows typical ferromagnetic behavior with a low coercive field of $\sim 5 \mathrm{kA} / \mathrm{m}$ and an expected saturation magnetization amounting to about $6.9 \mu_{\mathrm{B}} / \mathrm{Gd}$. On the other hand, the antiferromagnetic low temperature state of $\mathrm{GdGaH}_{1.66}$ is certified by Figure $7 \mathrm{~d}$ which shows a linear increase of the magnetization as a function of magnetic field at $6 \mathrm{~K}$. Because $\mathrm{GdGaH}_{1.66}$ easily desorbs $\mathrm{H}$, we checked and confirmed the integrity of the sample after the magnetization measurement by PXRD.

\section{Conclusions}

GdGa absorbs hydrogen at slightly elevated temperatures. Structural analysis by a combination of X-Ray powder diffraction and DFT modeling suggests that conditions with hydrogen pressures above 15 bar yield $\mathrm{GdGaH}_{1.66}$ with the $\mathrm{NdGaH}_{1.66}$ structure. $\mathrm{GdGaH}_{1.66}$ desorbs $\mathrm{H}$ above $\mathrm{T}=200{ }^{\circ} \mathrm{C}$. Without the presence of hydrogen, hydrides $\mathrm{GdGaH}_{\mathrm{x}}$ decompose at temperatures above $300{ }^{\circ} \mathrm{C}$ into $\mathrm{GdH}_{2}$ and an unidentified $\mathrm{Gd}-\mathrm{Ga}$ intermetallics. Thus the hydrogenation of $\mathrm{GdGa}$ is not reversible. This is in contrast with the system $\mathrm{NdGa}$ $/ \mathrm{NdGaH}_{1.66}$ which exhibits reversible $\mathrm{H}$ storage capability [20]. Hydrogenation induces a change in the magnetic properties: coupling changes from being ferromagnetic in GdGa to antiferromagentic in $\mathrm{GdGaH}_{1.66}$. The effective number of bohr magnetons is close to that of GdGa above Tc, indicating that Gd possesses the same oxidation state $(+3)$ in both systems.

Acknowledgement. This work has been supported by the Swedish research council through grants 2010-4827 and 2013-4690.

\section{Appendix A. Supporting information}

Supplementary data associated with this article can be found in the online version at XXXXX 


\section{References}

[1] O. Schob, E. Parthé, Acta Crystallogr. 19 (1965) 214-224.

[2] A. E. Dwight, J. W. Downey, R. A. Conner, Acta Crystallogr. 23 (1967) 860-862.

[3] P. Villars, L. D. Calvert, Pearson's Handbook of Crystallographic Data for Intermetallic Compounds, Sec. Ed., ASM International: Materials Park, OH, 1991, and Desk Edition, 1997.

[4] J. Y. Zhang, J. Luo, J.B. Li, J. K. Liang, Y. C. Wang, L. N. Ji, J. Alloy Compd. 469 (2009) $15-19$.

[5] J. Chen, B. G. Shen, Q. Y. Dong, F. X. Hu, J. R. Sun, Appl. Phys. Lett. 95 (2009) 132504.

[6] J. Chen, B. G. Shen, Q. Y. Dong, J. R. Sun, Solid State Commun. 150 (2010) 157-159.

[7] X. Q. Zheng, J. Chen, J. Shen, H. Zhang, Z. Y. Xu, W. W. Gao, J. Appl. Phys. 111 (2012) $07 \mathrm{~A} 917$.

[8] T. Gao, K. Nishimura, T. Matsumoto, T. Namiki, Y. Isikawa, Solid State Commun. 158 (2013) $1-4$.

[9] Z.-J. Mo, J. Shen, L.-Q. Yan, C.-C. Tang, J. Lin, J.-F. Wu, Appl. Phys. Lett. 103 (2013) 052409.

[10] X. Q. Zheng, J. Chen, L. C. Wang, R. R. Wu, F. X. Hu, J. R. Sun, J. Appl. Phys. 115 (2014) 17A905.

[11] B. Barbara, V. N. Nyugen, E. Siaud, C.R. Acad. Sci. B. 274 (1972) 1053-1056.

[12] H. Fujii, N. Shohata, T. Okamoto, E. Tatsumoto, J. Phys. Soc. Jpn. 31 (1971) 15921592.

[13] N. Shohata, J. Phys. Soc. Jpn. 42 (1977) 1873-1880.

[14] N. N. Delyagin, V. I. Krylov, I. N. Rozantsev, J. Magn. Magn. Mater. 308 (2007) 74-79.

[15] (a) M. A. Ruderman, C. Kittel, Phys. Rev. 96 (1954), 99-102. (b) T. Kasuya, Prog. Theor. Phys. 16 (1956), 45-57. (c) K. Yosida, Phys. Rev. 106 (1957), 893-898.

[16] X. B. Liu, Z. Altounian, Physica B 406 (2011) 710-714.

[17] S. C. Sevov, Zintl Phases, in: J. H. Westbrook, R. L. Fleischer (Eds.), Intermetallic Compounds - Principles and Practice, John Wiley \& Sons, Ltd, (2002)113-132.

[18] U. Häussermann, V. F. Kranak, K. Puhakainen, Hydrogenous Zintl Phases: Interstitial Versus Polyanionic Hydrides, in: T. F. Fässler (Ed.), Zintl Phases, Springer Berlin Heidelberg, 139 (2011)143-161.

[19] U. Häussermann, Z. Kristallogr. 223 (2008) 628-635. 
[20] J. Ångström, R. Johansson, T. Sarkar, M. H. Sørby, C. Zlotea, M. S. Andersson, Inorg. Chem. 55 (2016) 345-352.

[21] H. M. Rietveld, J. Appl. Crystallogr. 2 (1969) 65-71.

[22] (a) J. Rodriguez-Carvajal, J. FullProf 2.k, version 5.20; ILL: Grenoble, France, 2011; Rodríguez-Carvajal, J. Abstracts of the Satellite Meeting on Powder Diffraction of the XV IUCr Congress, (1990) 127. (b) J. Rodriguez-Carvajal. Int. Union Crystallogr. Newsl. 26 (2001) 12-19.

[23] Y. Cerenius, K. Ståhl, L. A. Svensson, T. Ursby, Å. Oskarsson, J. Albertsson, A. Liljas. J. Synchr. Radiat. 7 (2000) 203-208.

[24] T. R. Jensen, T. K. Nielsen, Y. Filinchuk, J.-E. Jørgensen, Y. Cerenius, E. M. Gray, J. Appl. Crystallogr. 43 (2010) 1456-1463.

[25] V. Höglin, J. Ångström, M. S. Andersson, O. Balmes, P. Nordblad, M. Sahlberg, Res. Phys. 5 (2015) 53-54.

[26] A. P. Hammersley, S. O. Svensson, M. Hanfland, A. N. Fitch, D. Häusermann, High Pressure Res. 14 (1996) 235-248.

[27] P. E. Blöchl, Phys. Rev. B. 50 (1994) 17953-17979.

[28] G. Kresse, D. Joubert, Phys. Rev. B. 59 (1999) 1758-1775.

[29] (a) G. Kresse, J. Hafner, Phys. Rev. B. 47 (1993) 558-561. (b) G. Kresse, J. Hafner, Phys. Rev. B. 49 (1994) 14251-14269.

[30] (a) G. Kresse, J. Furthmüller, Comp Mater Sci. 6 (1996) 15-50. (b) G. Kresse, J. Furthmüller, Phys. Rev. B. 54 (1996) 11169-11186.

[31] (a) J. P. Perdew, K. Burke, M. Ernzerhof, Phys. Rev. Lett. 77 (1996) 3865-3868. (b) J. P. Perdew, K. Burke, M. Ernzerhof, Phys. Rev. Lett. 78 (1997) 1396-1396.

[32] H. T. Stokes, D. M. Hatch, J. Appl. Crystallogr. 38 (2005) 237-238.

[33] H. J. Monkhorst, J. D. Pack, Phys. Rev. B. 13 (1976) 5188-5192.

[34] A. Leithe-Jasper, K. Hiebl, Phys. Status Solidi A 155 (1996) 223-231.

[35] R. A. Susilo, J. M. Cadogan, D. H. Ryan, N. R. Lee-Hone, R. Cobas, S. Muñoz-Pérez, Hyperfine Interact. 226 (2013) 257-266. 


\section{Figures}

\section{Figure 1}

a)

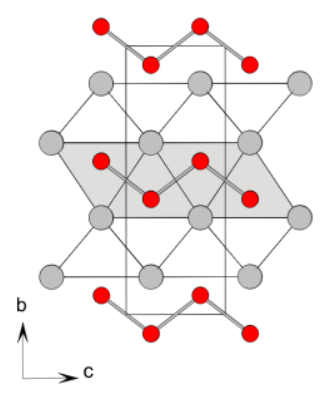

REGa

b) $\mathrm{NdGaH}_{1.66}$
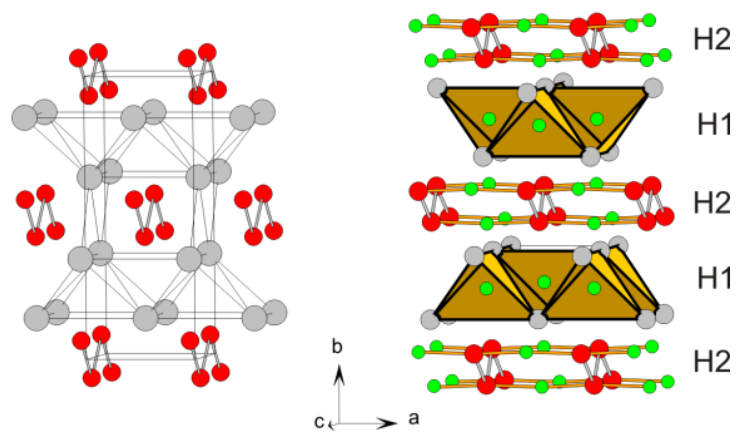

a) Cmcm CrB-type structure of rare earth (RE) monogallides. RE and Ga atoms are depicted as grey and red circles, respectively. RE atoms are arranged as slabs of trigonal prisms hosting linear zigzag chains of $\mathrm{Ga}$ atoms. b) The crystal structure of $\mathrm{NdGaD}_{1.66} . \mathrm{H}$ atoms are depicted as green circles. Stacking of slabs of $\mathrm{RE}_{6}$ trigonal prisms in the $\mathrm{CrB}$ structure produces layers of edge sharing tetrahedra which are centered by $\mathrm{H}(\mathrm{H} 1)$ atoms. Additional $\mathrm{H}$ atoms $(\mathrm{H} 2$, not fully occupied) are inserted between Ga atoms which results in quasi linear fragmented chains -Ga-H2-. 


\section{Figure 2}

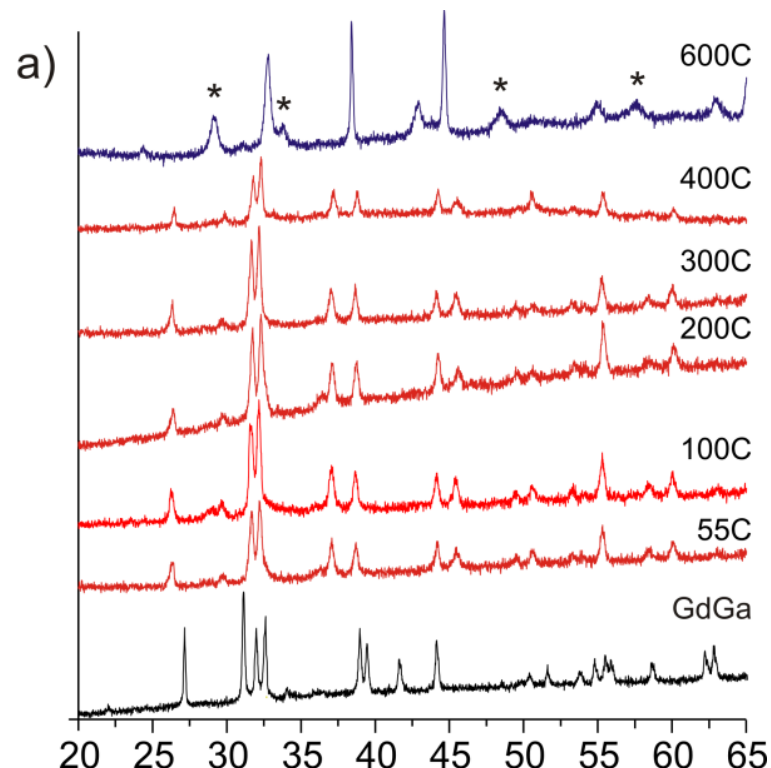

b)

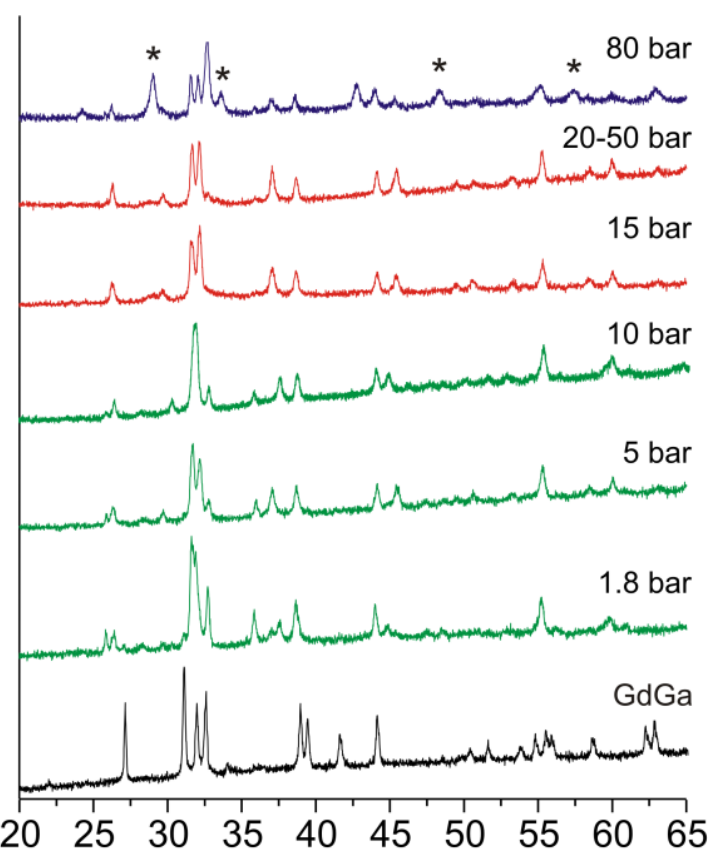

\section{2-Theta}

PXRD patterns $(\lambda=1.5418 \AA$ ) of products from the hydrogenation of GdGa at (a) p = 20 bar and over a $\mathrm{T}$ range of $50-600{ }^{\circ} \mathrm{C}$, (b) at $\mathrm{T}=100{ }^{\circ} \mathrm{C}$ and over a $\mathrm{p}$ range of $1.5-80 \mathrm{bar}$. Dwelling time was $2 \mathrm{~h}$ for all experiments. The pattern of the GdGa starting material is shown as black line. Red patterns: hydride phase $\mathrm{GdGaH}_{1.66}$; green patterns: intermediate hydride phase; blue patterns: decomposed products (reflections from $\mathrm{GdH}_{2}$ are marked with asterisks). 


\section{Figure 3}
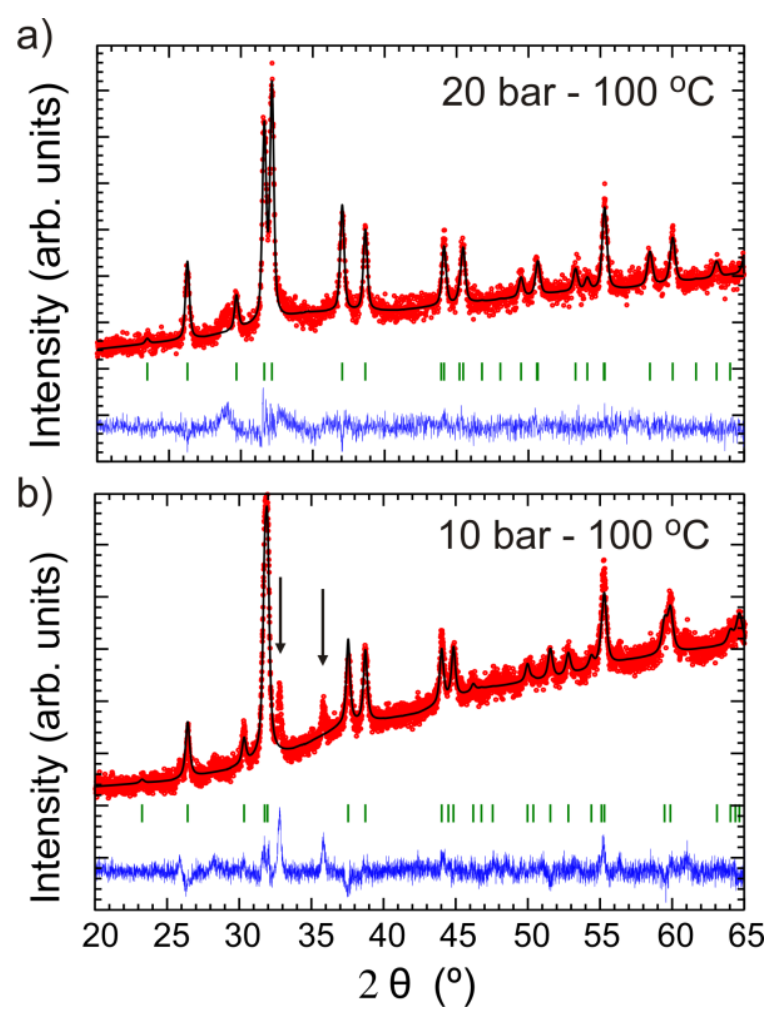

Rietveld fits to the PXRD data of hydrogenous GdGa samples obtained from annealing GdGa

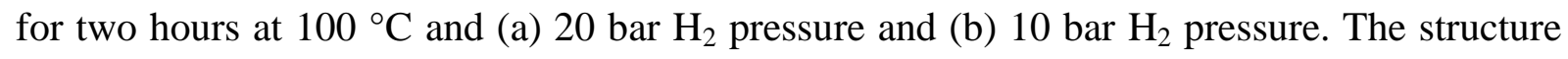
model of $\mathrm{NdGaH}_{1.66}$ can be fit satisfactorily to the data of the sample obtained at 20 bar. When applying it to the data of the sample obtained at 10 bar ("intermediate hydride phase", cf. Figure $2 b$ ) unindexed peaks remain (the most prominent ones are marked by arrows), which may be accounted for by a $2 \times a$ or $2 \times c$ supercell of the $\mathrm{NdGaH}_{1.66}(\mathrm{Cmcm})$ structure. 


\section{Figure 4}

a)

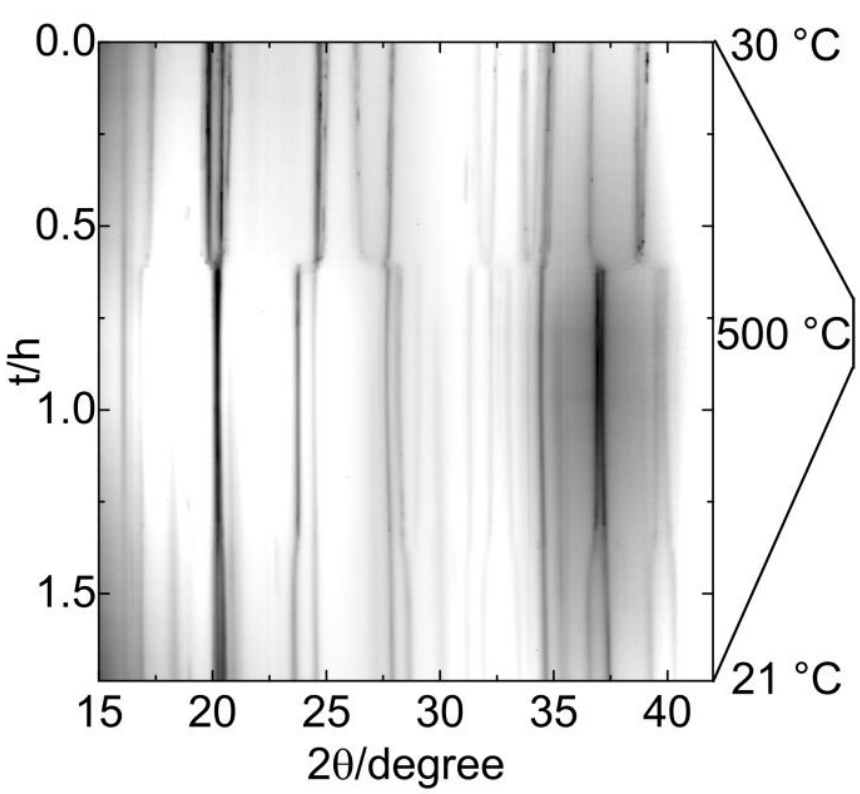

b)

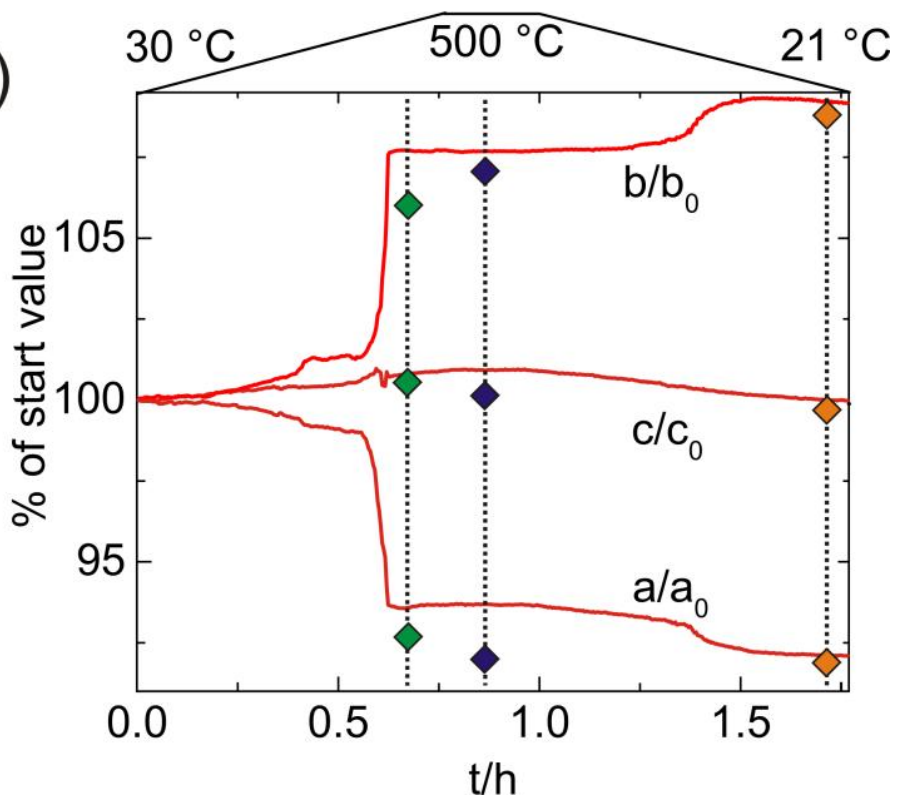

a) Densitometric view of the in-situ PXRD patterns ( $\lambda=0.991 \AA$ ) recorded during heating and cooling of $\mathrm{GdGa}$ using 20 bar $\mathrm{H}_{2}$ pressure. b) Relative change of lattice parameters as a function of time (red). DFT-calculated changes for $\mathrm{GdGaH}, \mathrm{GdGaH}_{1.33}$ and $\mathrm{GdGaH}_{1.66}$ are inset as green, blue, and orange symbols, respectively. 
Figure 5

a)
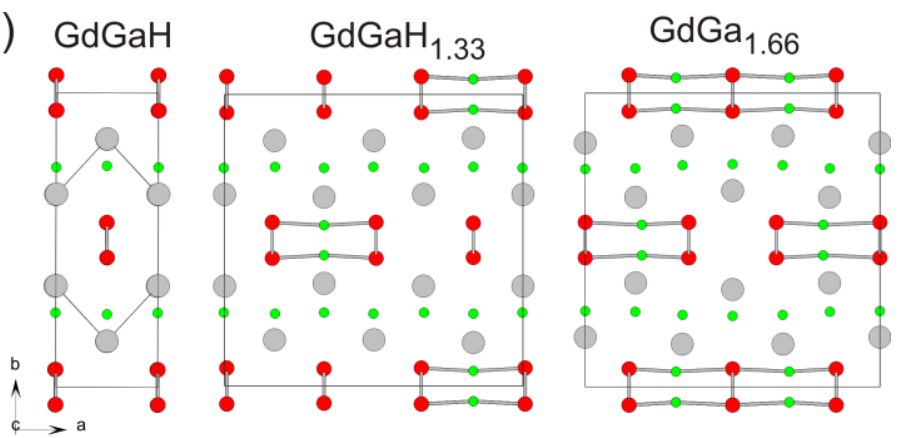

b)
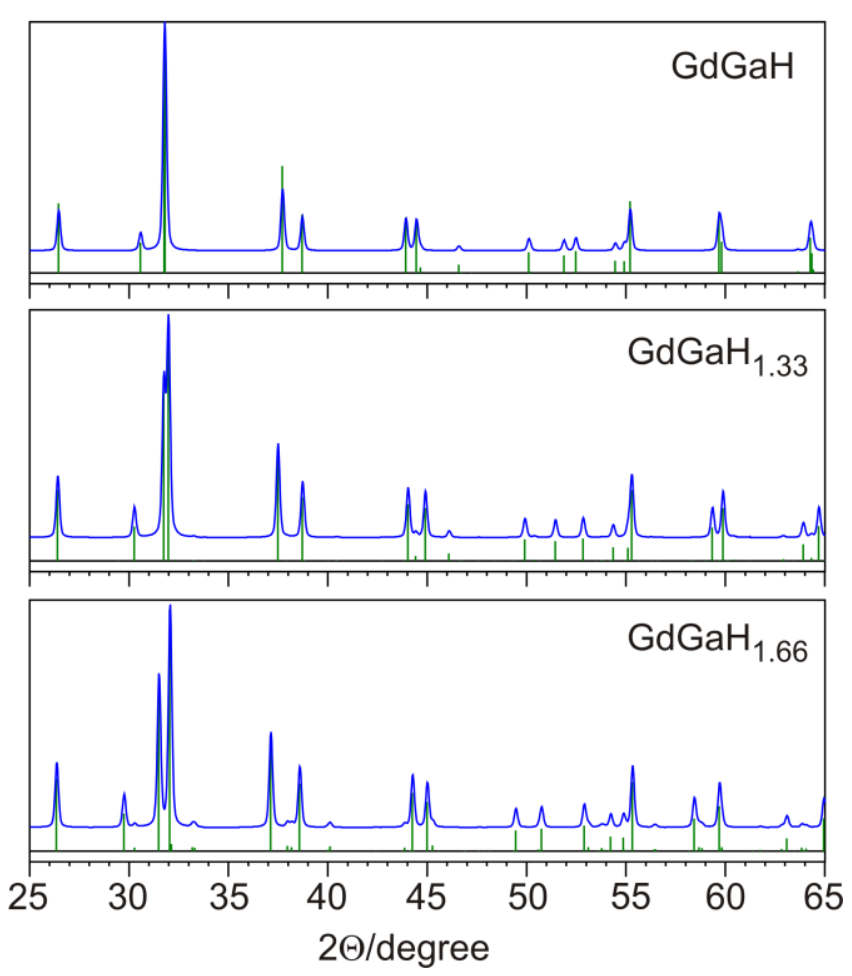

Compilation of DFT relaxed structures for $\mathrm{GdGaH}_{\mathrm{x}}$ (a) and corresponding powder patterns (b). The calculated patterns for $\mathrm{GdGaH}_{1.66}$ and $\mathrm{GdGaH}_{1.33}$ relate to the experimental PXRD patterns shown in Figure 3a and 3b, respectively. 


\section{Figure 6}

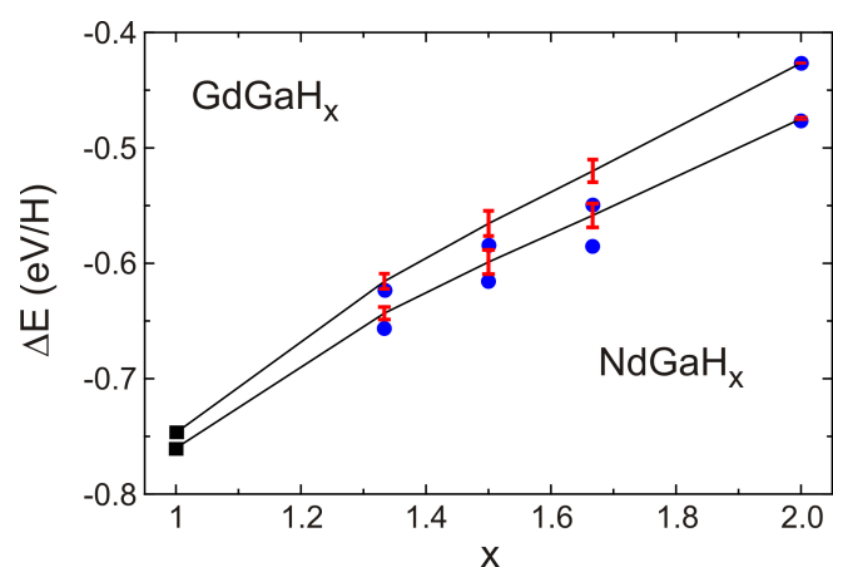

Heat of formations REGa $+\mathrm{x} / 2 \mathrm{H}_{2}=\mathrm{REGaH}_{\mathrm{x}}$ as a function of $\mathrm{x} .(\mathrm{RE}=\mathrm{Gd}$ (upper line) and $\mathrm{Nd}$ (lower line)). $\mathrm{x}=1.0$ corresponds to $\mathrm{REGaH}(\mathrm{Z}=4$ cell). $\mathrm{x}=1.33,1.5,1.66,2.0$ represent results from $3 \times$ a supercell $(Z=12)$ calculations. The line connects average values from 50 random starting configurations, and the red bars indicate the standard deviation. Blue symbols represent the obtained lowest energy values.

\section{Figure 7}
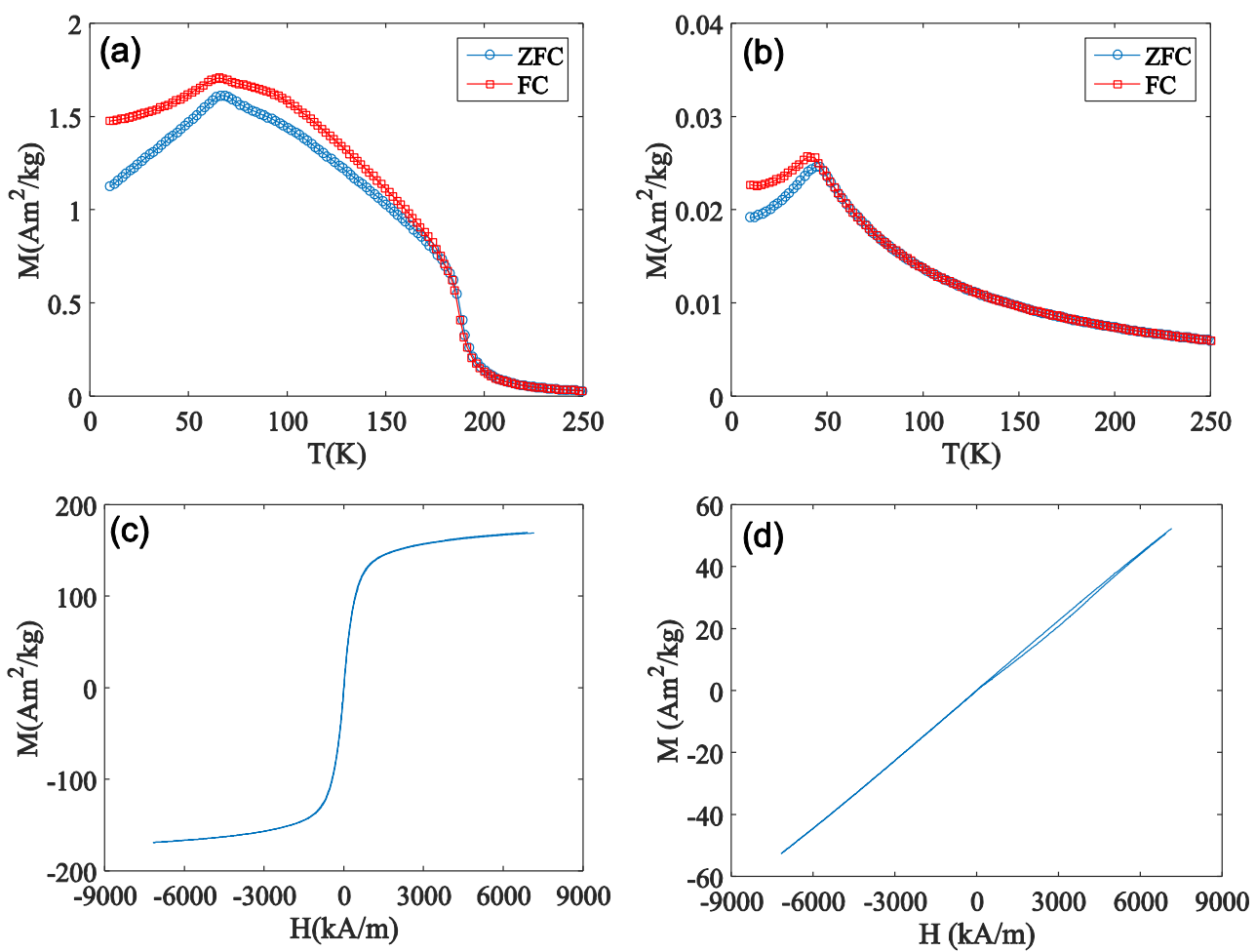

Top: Zero field cooled $\mathrm{M} v s \mathrm{~T}$ curves of (a) $\mathrm{GdGa}$ and (b) $\mathrm{GdGa}(\mathrm{H})_{1.66}$ at $\mathrm{H}=4 \mathrm{kA} / \mathrm{m}$. Bottom: $\mathrm{M}$ vs $\mathrm{H}$ curves at $\mathrm{T}=6 \mathrm{~K}$ for $(\mathrm{c}) \mathrm{GdGa}$ and (d) $\mathrm{GdGa}(\mathrm{H})_{1.66}$ 
Tables:

Table 1. Crystallographic data and structure refinement for $\mathrm{GdGa}$ and $\mathrm{GdGaH}_{1.66}$ from PXRD data $(\mathrm{T}=295 \mathrm{~K})$

\begin{tabular}{|c|c|c|c|}
\hline empirical formula & GdGa & $\begin{array}{c}\mathrm{GdGaH}_{1.66} \\
20 \mathrm{bar} / 100{ }^{\circ} \mathrm{C}\end{array}$ & $\begin{array}{c}\mathrm{GdGaH}_{\mathrm{x}} \\
10 \mathrm{bar} / 100{ }^{\circ} \mathrm{C}\end{array}$ \\
\hline space group & $C m c m$ & $C m c m$ & $C m c m$ \\
\hline unit cell $(\AA)$ & $\begin{array}{c}a=4.340(3) \\
b=11.012(2) \\
c=4.105(3)\end{array}$ & $\begin{array}{c}a=3.9867(7) \\
b=12.024(2) \\
c=4.1009(6)\end{array}$ & $\begin{array}{c}a=4.038(7) \\
b=11.77(2) \\
c=4.109(6)\end{array}$ \\
\hline $\mathrm{Gd} 4 \mathrm{c}(0, \mathrm{y}, 1 / 4)$ & $0.1414(3)$ & $0.151(2)$ & $0.1509(3)$ \\
\hline $\mathrm{Ga} 4 \mathrm{c}(0, \mathrm{y}, 1 / 4)$ & $0.4245(5)$ & $0.445(5)$ & $0.4457(7)$ \\
\hline$V\left(\AA^{3}\right)$ & $196.2(4)$ & $196.6(5)$ & $195.4(5)$ \\
\hline $\mathrm{R}_{\mathrm{f}}$ & 2.05 & 2.40 & 2.47 \\
\hline $\mathrm{R}_{\mathrm{wp}}$ & 2.65 & 3.06 & 3.39 \\
\hline
\end{tabular}

Table 2. Lattice parameters and unit cell volumes of DFT optimized structures $\mathrm{GdGaH}_{\mathrm{x}}$. Relative changes (in \%) with respect to $\mathrm{GdGa}(\mathrm{x}=0)$ are given in parentheses. (See also Table S1, supporting information)

\begin{tabular}{|l|l|l|l|l|}
\hline composition & $a(\AA)$ & $b(\AA)$ & $c(\AA)$ & $V\left(\AA^{3}\right)$ \\
\hline 0 & 4.3891 & 11.0250 & 4.0970 & 198.3 \\
\hline 1 & $4.0728(-7.2)$ & $11.6885(+6.0)$ & $4.1199(+0.5)$ & $196.1(-1.1)$ \\
\hline 1.33 & $4.0346(-8.1)$ & $11.8071(+7.1)$ & $4.1105(+0.3)$ & $195.8(-1.2)$ \\
\hline 1.50 & $4.0298(-8.2)$ & $11.9021(+8.0)$ & $4.1022(+0.1)$ & $196.8(-0.8)$ \\
\hline 1.66 & $4.027(-8.3)$ & $12.0092(+8.9)$ & $4.0906(-0.2)$ & $197.8(-0.2)$ \\
\hline 2 & $4.1023(-6.5)$ & $11.8529(+7.5)$ & $4.0834(-0.3)$ & $198.5(+0.1)$ \\
\hline
\end{tabular}


Table 3. Effective number of bohr magnetons ( $\left.\mu_{\text {eff }}\right)$ and Curie-Weiss constants $\left(\theta_{\mathrm{CW}}\right)$ obtained from Curie-Weiss fits to the high temperature susceptibility data.

\begin{tabular}{|l|l|l|}
\hline Sample & $\mu_{\text {eff }}$ & $\theta_{\mathrm{CW}}(\mathrm{K})$ \\
\hline GdGa & 8.4 & 190 \\
\hline GdGaH $_{1.66}$ & 7.9 & -13 \\
\hline
\end{tabular}




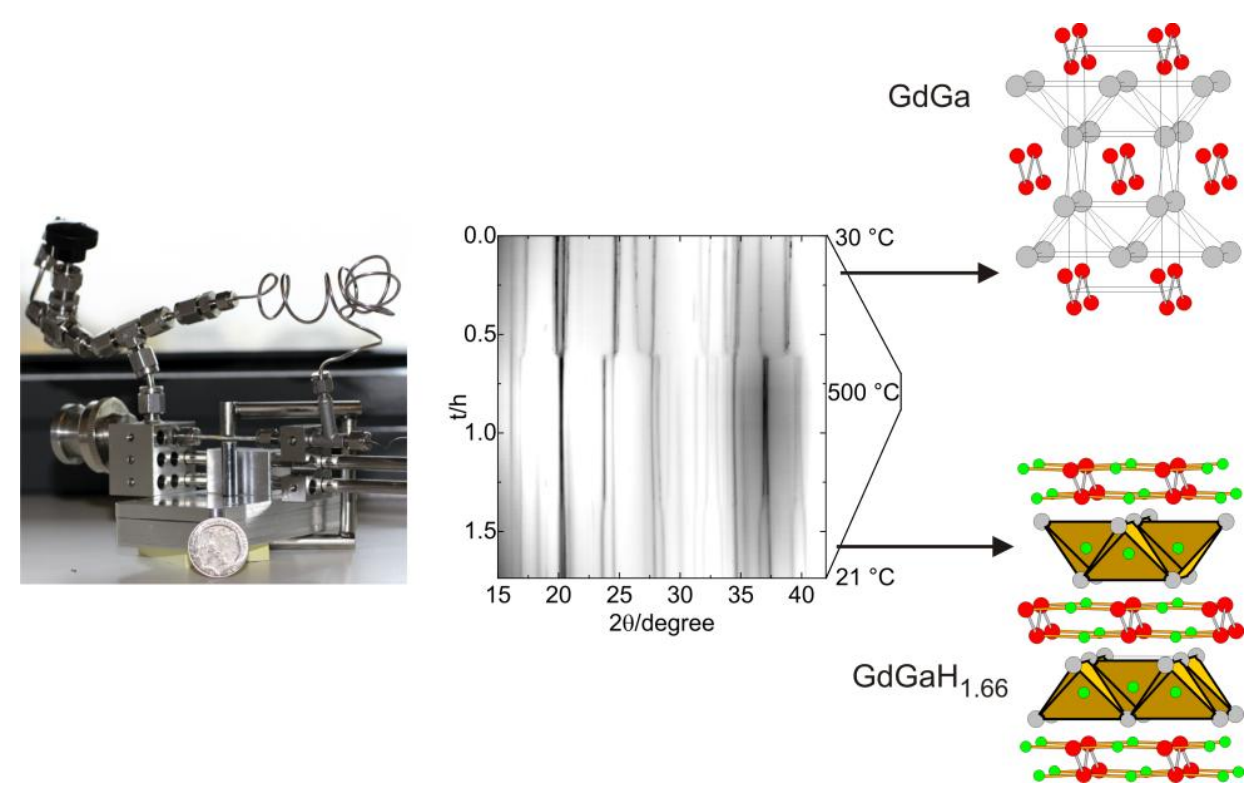

Ferromagnetic GdGa absorbs hydrogen in two steps to yield antiferromagnetic $\mathrm{GdGaH}_{1.66}$. 


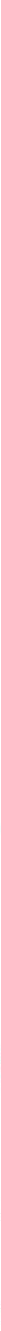

